\title{
Poliopolis: pushing boundaries of scientific innovations for disease eradication
}

Pierre Van Damme ${ }^{* \neq, 1}$, Ilse De Coster ${ }^{\ddagger}, 1$, Ananda S Bandyopadhyay ${ }^{2}$, Leen Suykens ${ }^{1}$, Patrick Rudelsheim ${ }^{3}$, Pieter Neels ${ }^{4}$, M Steven Oberste ${ }^{5}$, William C Weldon ${ }^{5}$, Ralf Clemens ${ }^{6}$ \&

Hilde Revets ${ }^{1}$

${ }^{1}$ Centre for the Evaluation of Vaccination, Vaccine \& Infectious Disease Institute, University of Antwerp, Belgium

${ }^{2}$ Bill \& Melinda Gates Foundation, Seattle, WA, USA

${ }^{3}$ Biosafety coordinator University of Antwerp, Perseus BVBA, Belgium

${ }^{4}$ Vaccine Advice BVBA, Zoersel, Belgium

${ }^{5}$ Centers for Disease Control \& Prevention, Atlanta, GA, USA

${ }^{6}$ Global Research in Infectious Diseases (GRID), Rio de Janeiro, Brazil

*Author for correspondence: pierre.vandamme@uantwerpen.be

$\ddagger$ Authors contributed equally

Although global polio eradication is within reach, sustained eradication of all polioviruses requires cessation of oral poliovirus vaccine use to mitigate against vaccine-derived poliovirus circulation and vaccineassociated paralytic poliomyelitis. The first step in this direction was the WHO-recommended global withdrawal of live attenuated type 2 Sabin poliovirus from routine immunisation in May 2016, with future use restricted to outbreak response, and handling controlled by strict containment provisions (GAPIII). This creates unique challenges for development and testing of novel type 2 poliovirus vaccines. We describe the creation of a novel purpose-built containment facility, Poliopolis, to study new monovalent OPV2 vaccine candidates in healthy adult volunteers, which may be a model for future endeavors in vaccine development for emergency use.

First draft submitted: 26 June 2019; Accepted for publication: 20 August 2019; Published online: 4 September 2019

Keywords: containment $\bullet$ eradication $\bullet \mathrm{OPV} \bullet$ oral $\bullet$ poliomyelitis $\bullet$ poliovirus $\bullet$ psychological screening $\bullet$ purposebuilt facility $\bullet$ vaccine $\bullet$ volunteers

Poliomyelitis is close to becoming the second vaccine-preventable disease, after smallpox, ever to be eradicated. In 2015, wild type 2 poliovirus (WPV2) was certified eradicated after the last naturally occurring case was reported in 1999. Wild type 3 poliovirus (WPV3) transmission has also likely been interrupted with no case or environmental isolation since 2012 [1,2]. Three countries, Nigeria, Pakistan and Afghanistan remain endemic for transmission of wild type 1 poliovirus (WPV1) [2], although a silent outbreak of WPV1 was detected in Israel in 2013-2014, but no clinical cases were reported [3]. The Sabin live attenuated trivalent oral poliovirus vaccine (OPV) has played a major role in interrupting polio transmission globally, in rare circumstances it can revert to neurovirulence resulting in vaccine-associated paralytic poliomyelitis in vaccinees or their close contacts [4]. In settings of low population immunity due to poor immunization coverage, excreted OPV strains can also acquire neurovirulence and transmissibility, leading to circulating vaccine-derived polioviruses (cVDPV). The risk of cVDPV spread in polio-free countries has been illustrated in the past two decades, with outbreaks reported from the Caribbean, Asia and Africa and most recently, in situations of social breakdown, security and conflict issues such as in Ukraine, Syria and Iraq, and Nigeria and Somalia [5-13], and most recently in Papua New Guinea [14]. A particular concern is the transmission of polioviruses including cVDPV from outbreaks in such regions into neighboring countries and beyond [15,16]. 


\section{The need for novel polio vaccines}

Polio eradication will not be complete unless the risks of spread and transmission from all types of polioviruses, including VDPV, are adequately mitigated. The endgame of polio eradication, therefore, has complex vaccine choices. Trivalent inactivated polio vaccine (IPV) induces excellent humoural immunogenicity and thereby prevents paralysis, but its impact on intestinal immunity - and as such on transmission - is limited compared with OPV [1719]. In settings of poor sanitation and hygiene where the fecal-oral route of transmission predominates, OPV is a more effective vaccine to interrupt person-to-person transmission, and thus has typically been the vaccine-of-choice for outbreak response. However, the risks of vaccine-associated paralytic poliomyelitis and VDPV arising from OPV usage, although rare [20], are an important consideration in the context of achieving and sustaining complete eradication of polio. With the global cessation of all Sabin type 2 vaccine use, intestinal immunity to type 2 is also on the decline. Under these circumstances, in the event of a vaccine-derived type 2 polio outbreak, the use of the current stockpiled monovalent OPV2 is the only option for outbreak control but brings with it its own risk of generating new type 2 cVDPVs.

To minimize this risk and to ensure complete eradication, a scientific consortium supported by the Bill \& Melinda Gates Foundation in coordination with vaccine developers and global agencies (WHO, PATH) has developed novel OPV vaccine strains with the intent of stockpiling such vaccines for emergency use, if and when needed for outbreak response [21,22]. The genetic sites of attenuation have been identified and the genetic sequence can be manipulated to stabilise these attenuations and minimize the frequency of reversion. Two novel OPV type 2 (nOPV2) candidates based on attenuated serotype 2 polioviruses derived from a modified Sabin 2 infectious cDNA clone have been genetically designed, engineered, produced and tested in a series of preclinical studies [21,23]. In these preclinical models, the two candidates have been proven to be less transmissible and genetically more stable than the Sabin OPV, and so less likely to revert to neurovirulent strains that are shed in vaccinees' stools. The natural consequence of these preclinical studies was the proposal to test the two candidates in human volunteers in a Phase I study.

Given the current unique situation of global certification of WPV2 eradication, the subsequent global cessation of all elective use of OPV containing type 2 from May 2016, and the recommendations of the WHO Global Action Plan to minimize poliovirus facility-associated risk after type-specific eradication of wild polioviruses and sequential cessation of OPV use (GAPIII) [24], studies with new live polio vaccines require specific containment measures [25]. In this paper we describe the implementation of a unique project that allowed the first-in-human Phase I clinical study with two attenuated nOPV2 candidates to evaluate these novel vaccines in healthy adult volunteers in these unprecedented circumstances of containment. The study was performed with extensive monitoring and with the time pressure in the current context of multiple and increasing type 2 cVDPV outbreaks taking place in different parts of the world. The encouraging results of that study have recently been reported [23].

\section{The rationale for containment}

The objectives of this first-in-human Phase I, blinded, single-center trial were the assessment of the safety and the immunogenicity of two nOPV2 candidates in healthy adult volunteers. The two nOPV2 candidates (S2/cre5/S15domV/rec1/hifi3 and S2/S15domV/CpG40) are live-attenuated serotype-2 polioviruses derived from a modified Sabin type-2 infectious cDNA clone propagated in Vero cells. The viral nucleotide sequences in parts of the $5^{\prime}$-untranslated region of both candidates are modified to improve the genetic stability of this major attenuating determinant of Sabin type-2, with various other modifications made to further improve stability of the attenuation, inhibit recombination and reduce replicative fitness to improve stability of the attenuated phenotype while also reducing transmission [23].

The trial also included extensive assessment of viral shedding in stool samples and testing of shed virus for genetic stability and neurovirulence following oral receipt of one of the two nOPV2 candidates. In addition to containment recommendations (GAPIII), which currently apply to all type 2 polioviruses, the novelty of the genetically modified nOPV2 viruses necessitated performing the study in a fully contained environment with maximal effort to avoid any accidental release into the environment by ensuring that all biological samples that could potentially contain vaccine virus were captured and contained for subsequent decontamination.

Previous quarantine and human challenge studies reported isolation of clinical trial volunteers for 9-14 days [26,27]. In view of the nature of orally administered polioviruses and available shedding data, a longer quarantine period of 28 days was recommended for the study on nOPV2 candidates. Shedding data reported in a study in Panama of previously IPV-vaccinated children challenged with licensed OPV2 vaccine indicates that 1 week after challenge, 
$78.3 \%$ experience shedding with a median fecal titer of $4.45 \log \mathrm{CCID}_{50}(50 \%$ of the cell culture infectious dose), which dropped to $46 \%$ and $2.75 \log \mathrm{CCID}_{50}$, respectively after 3 weeks [18]. Across studies, 63-100\% of IPV-vaccinated children demonstrate fecal excretion at 7-10 days [19,28,29]. One recent study in 144 IPV-primed adults challenged with OPV1, showed that $98 \%$ were infected, at a peak stool titer of $10^{6.0} \mathrm{CCID}_{50} / \mathrm{gram}$ and shed the challenge virus for a mean of 13 days [26]. Thus, a novel purpose-built facility was created for sufficient numbers of study volunteers to be accommodated in isolation from the external environment for a period of 28 days, a unique situation for a vaccine trial.

\section{Planning}

The Center for the Evaluation of Vaccination (University of Antwerp, Belgium) was contacted by the Bill \& Melinda Gates Foundation in December 2016 with a request to perform a Phase I study with the nOPV2 candidates. To ensure the vaccine strains did not get into the environment through excretion of virus in stools from the vaccinated volunteers and potential manual transmission, the capture of all excreted fluids from the vaccinated volunteers and standard collection and disposal of clothing and all other materials handled by them, for example, towels, disposable eating utensils, uneaten food and all waste would have to be strictly enforced. As standard Phase I facilities are not designed to meet these specific requirements and after having screened alternative containment facilities like isolated holiday accommodations, unoccupied buildings (such as unused or empty closed centers for asylum seekers), together with the specificity of the study and the biosafety requirements, it was decided that the only option was to construct a new purpose-built quarantine facility. A geographical requirement was that the facility had to be located close to a hospital in case of any medical emergency, and in view of the participation of the personnel of the Center for the Evaluation of Vaccination, the Antwerp University Hospital was readily identified as the site-of-choice. Acknowledging that the facility would not be a permanent structure, the original intention being for a duration of 2 years, it was agreed that the University of Antwerp would construct a temporary self-contained unit. The transitory nature of the facility immediately suggested building it using purpose-built modular 'containers'.

As soon as conceptual plans for the construction were available the interactions with technical support services, biosafety experts and the local municipality were initiated. Other expert groups and authorities including the local police and fire brigade became involved later in the planning. In the subsequent 5 months, the environmental and building applications were submitted and approved, approvals from the Ethics Committee and Belgian Regulatory authorities were obtained and an intervention dossier for the fire brigade was finalized. One of the key challenges was to find technical and balanced solutions to the diverse and sometimes conflicting concerns of these groups, for example, easy access for the fire brigade while the police wanted limited access to ensure external safety and easy control of the area (particularly with the externally located effluent containment tanks). Further considerations were the requirements for the 'contained use' of genetically modified organisms [30] and the stringent restrictions for OPV2, when developing entry and exit procedures, emergency plans and waste and effluent treatment processes. The close collaboration that developed between the clinical trial team, the facility manager and the respective university and hospital (bio)safety officers to find workable technical solutions and procedures also formed the basis for submitting the necessary applications and obtaining the approvals from the competent authorities (including the specific regional biosafety notification for a contained use of a GMO, reference SBB 219 2017/0209K).

\section{Building the infrastructure, 'Poliopolis'}

Due to the proactive planning and early off-site manufacture of the prefabricated modules by the company who designed them, the facility, now named Poliopolis, was constructed over the course of 3 days in April 2017 and finalised within 1 month. This was exactly 5 months after the decision was taken to set up this study, during which period all necessary local and national building and scientific approvals were obtained. The final construction was a one-storey building composed of 66 specially designed and constructed linked modules that housed all facilities in a contained environment (Figure 1). Facilities included private, individual bedrooms for a maximum of 17 volunteers, a common kitchen and dining room, a recreation room with TV and library, a fitness room with gymnasium equipment, shower rooms and toilets (including separate toilets for study staff) and a laboratory facility for on-site testing and sample preparation. There were also offices for the clinical staff and the study psychologist to examine and interview participants and collect samples. In addition there was a room for decontamination of materials, and four separate entrances and exits (Figure 2) - namely, an entrance for staff where they put on protective clothing (overall, overshoes and gloves) and a separate exit for removal of said clothing - the two rooms 


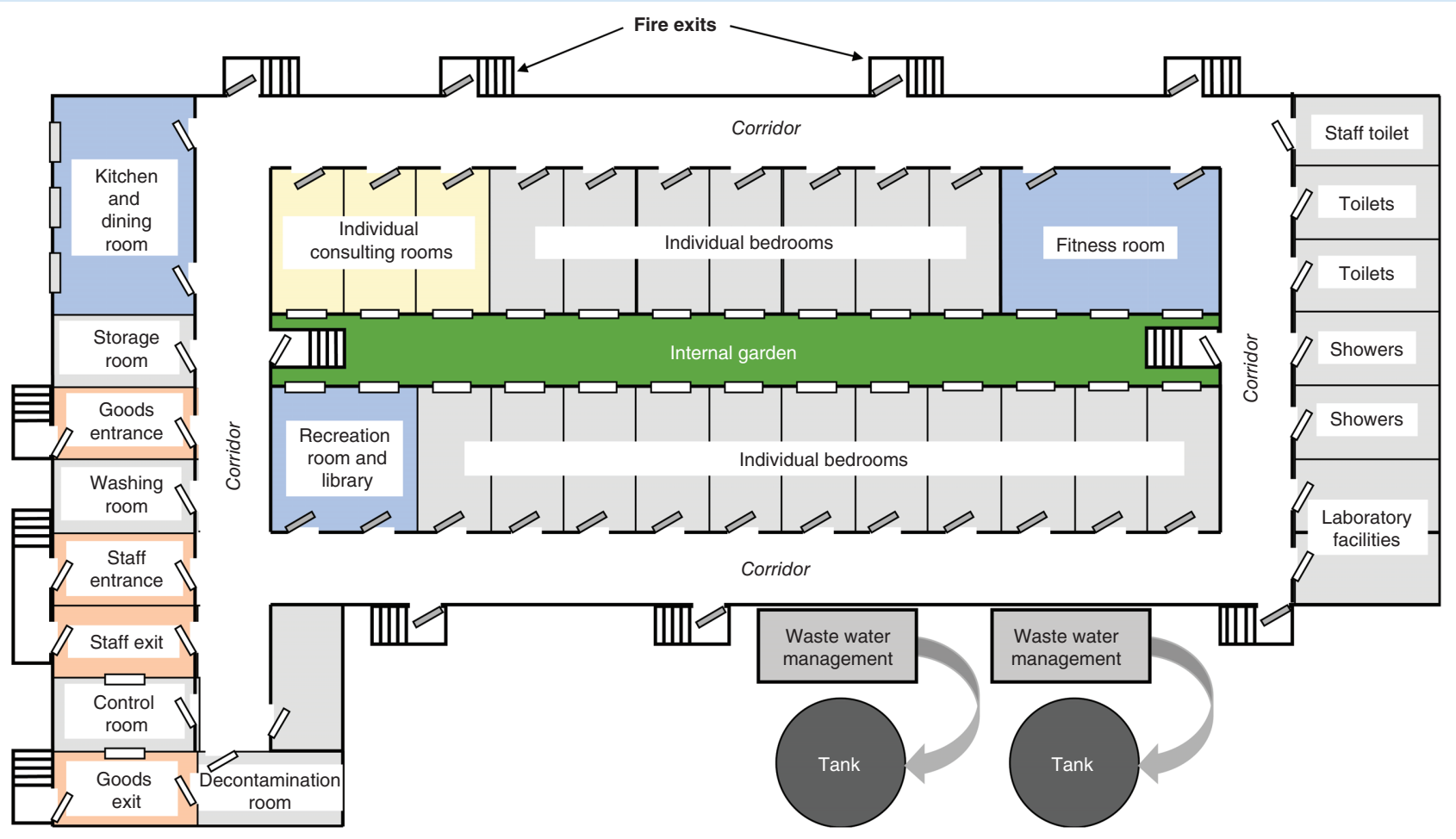

Figure 1. Diagrammatic representation of the modular design of the Poliopolis facility.

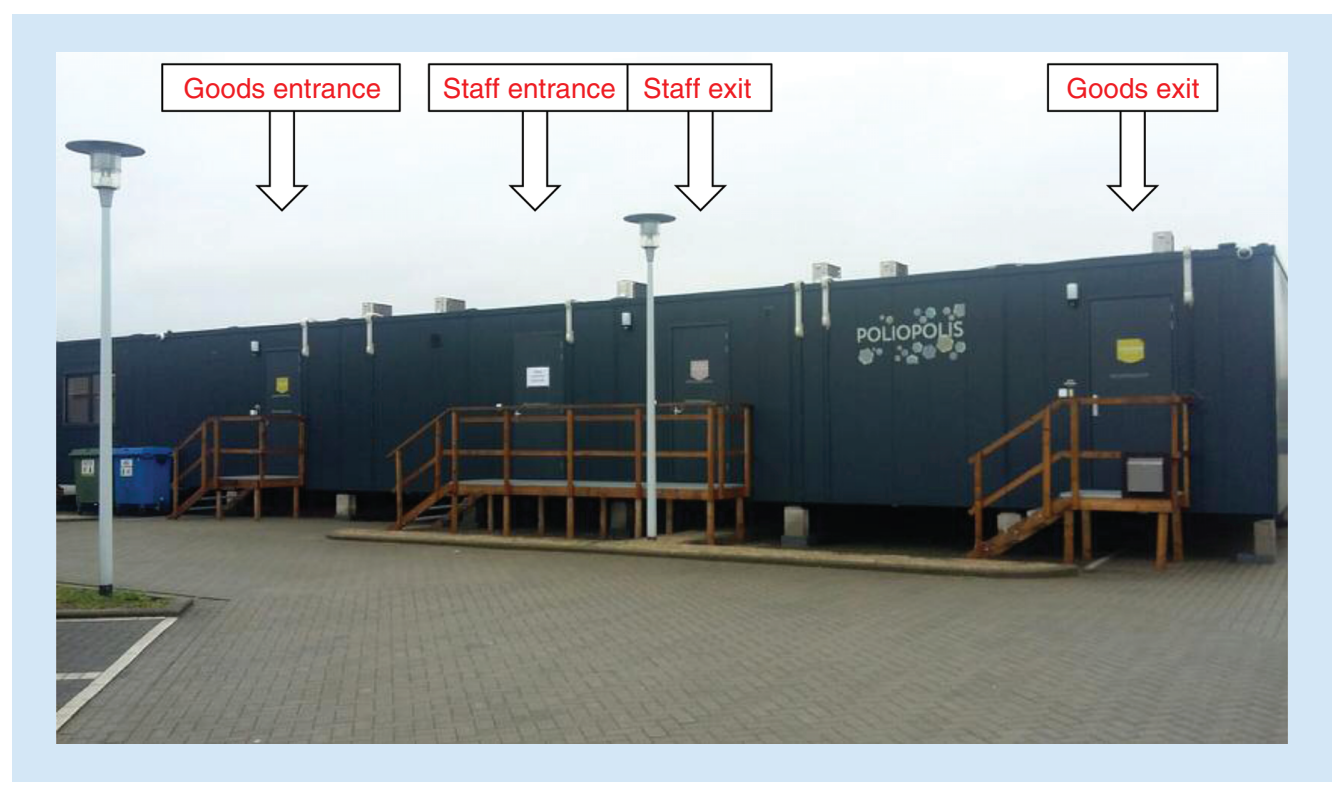

Figure 2. External appearance of Poliopolis showing the personnel entrance (left hand door) and exit (middle door) and the goods entrance and exit.

being connected by pass-through lockers where their outdoor clothing and personal belongings, laptops or cell phones were stored during their visit to the facility. The staff exit room also had a shower facility. In case clinical staff had been contaminated, for example, disruption of the overshoes, they had to take a shower of at least 5 min 
and redress with clean clothes that were kept available in that room. When the volunteers left the facility after 28 days, a similar procedure was followed for decontamination.

There was a separate entrance for receipt of goods (food, laboratory supplies, etc.) and an exit for the materials and goods leaving the facility after external decontamination (e.g., waste materials for incineration, stool and other samples from the participants). These were the only means of entry into the facility, although fire exits that could only be unlocked from the inside were also present. All entrances and exits were linked with alarm systems to ensure no unauthorised access in or out of the facility once the trial was underway. These entrances and exits worked as 'air locks', when the outside door was opened with a magnetic key, the inner door could not be opened until the outside door was closed again. This prevented anyone from entering or exiting the facility without being identified or dressed as required.

The kitchen was equipped with one-way glass windows allowing volunteers to look outside but preventing the public from seeing into the facility. Individual bedrooms and clinical offices had windows onto a central atrium which was open to the sky. This area was set up with garden furniture to allow participants to be outside, together with a barbecue facility for social events during the trial. Power and water were supplied directly from the hospital building, while the capture and containment of all waste meant that no sewage services were necessary.

All study personnel involved in direct interaction with the volunteers had to avoid contamination or accidental release of study vaccines or samples into the environment through use of body coverings (gowning), but not masks or eye protection. Since the risk of spread of vaccine virus through aerosols or droplets in the study population was considered negligible, air filtration of the facility and masking of the study personnel was not considered necessary, although masking and eye protection were recommended during medical visits when oropharyngeal swabs were taken.

The importance of wearing a gown was implicit to avoid any potential release of vaccine virus particles into the external environment on clothing of personnel leaving the contained facility, so training of personnel was critical to success. Hence, competency of gowning/de-gowning procedures in a 'green zone' was clearly documented, and periodic gowning certification conducted to confirm personnel maintained consistent practices. As with study personnel, all people who entered the facility were administered an IPV booster dose at least 14 days before study start and were trained in the gowning/de-gowning procedure by a qualified person.

A dedicated emergency team and vehicle was on constant stand-by, although never used to provide transport between the facility and the emergency room of the university hospital in the case of any medical emergency. As decontamination of the vehicle following transport of a vaccinated volunteer would take $3-4 \mathrm{~h}$, this precluded use of a standard emergency vehicle.

\section{External decontamination}

A special decontamination team was established and trained for a rapid, effective decontamination response according to a dedicated standard operating procedure (SOP) describing an emergency response plan, including an incident command system for emergency responses.

As noted, all wastewater was collected for subsequent decontamination, which included not only water from toilets, but also from showers, wash basins in bathrooms and kitchens and clothes-washing facilities. Two doublewalled 20,000 1 capacity tanks were set up the outside of the facility (Figure 3) for collection and storage of wastewater, for subsequent collection and decontamination by a specialist company.

A two-pronged approach was chosen to ensure adequate disinfection of the collected wastewater, initially using chlorine dioxide treatment followed by a $\mathrm{pH}$ increase through addition of sodium hydroxide prior to discharge in a municipal wastewater treatment plant. Chlorine dioxide was selected as disinfectant based on several scientific studies showing high efficacy of the active substance in killing poliovirus in wastewater [31-33]. One of these studies indicated that approximately $5 \log _{10}$ killing (i.e. $>99.999 \%$ reduction) was reached with a dose of $5 \mathrm{mg} / 1$ [33]. To ensure equivalent or more disinfection efficacy, a dose of $90 \mathrm{mg} / \mathrm{l}$ was used for decontamination of the wastewater.

For decontamination of the facility, chlorine dioxide gas was used. In addition to its high efficacy in killing poliovirus, chlorine dioxide gas has the added benefit of being smaller than all microorganisms, with a molecular size of $0.124 \mathrm{~nm}$, so no organism can be concealed from the gas. Chlorine dioxide gas can be accurately measured in real time from multiple points within the area being decontaminated, guaranteeing that the correct dosage needed for an effective decontamination is being met before the decontamination is deemed complete and aeration is started. The chlorine dioxide product used was Diox Forte $0.75 \%$, which contains $7.5 \mathrm{~g} / \mathrm{l}$ chlorine dioxide and is registered as a sterilant capable of eliminating all viruses, bacteria, fungi and spores. This product, among others, 


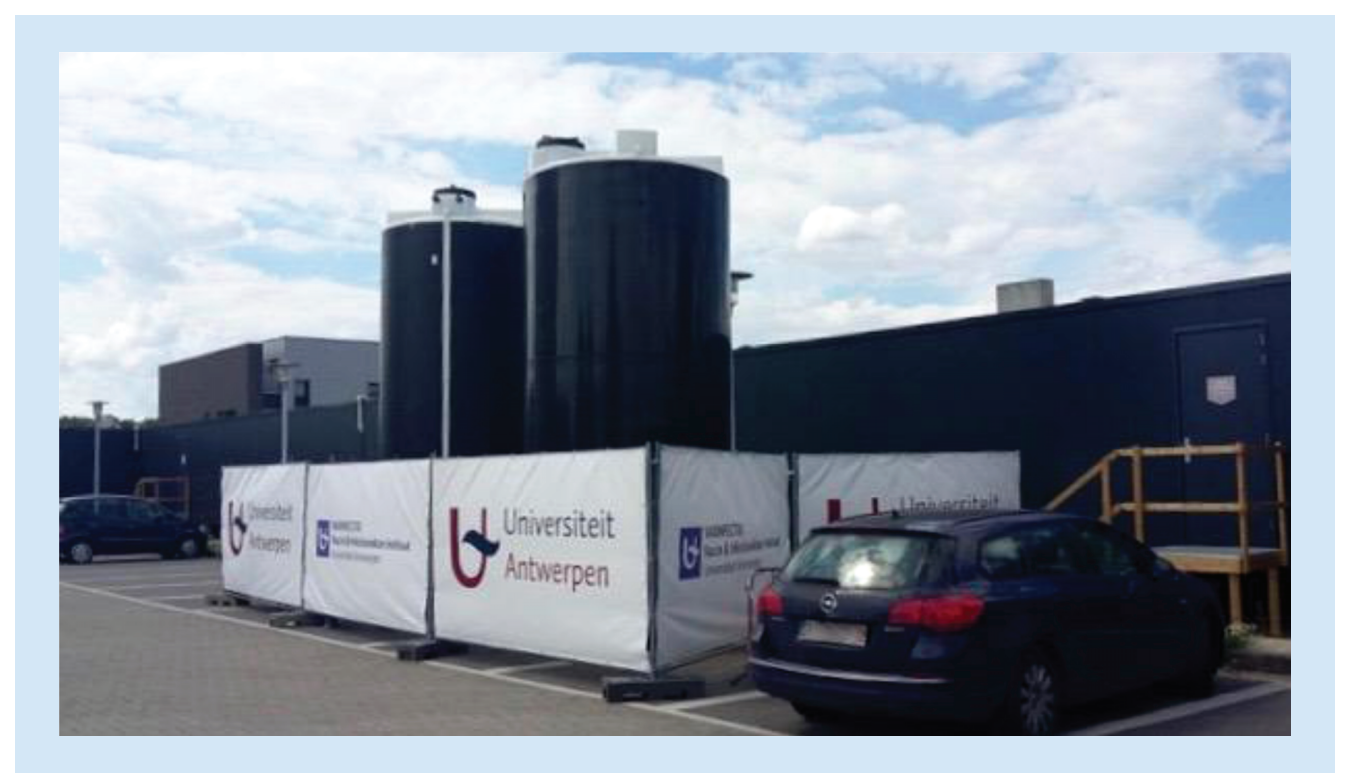

Figure 3. External appearance of Poliopolis showing the two external waste water tanks.

is approved for water disinfection in Belgium. A WHO report on the thermostability of vaccines reports a strong decrease in the stability of OPV as the $\mathrm{pH}$ increases above 8 [34]. Thus, increasing the $\mathrm{pH}$ was added as another disinfection step to prevent contamination by contact with the wastewater outside the contained facility.

Discharge of the decontaminated wastewater into a municipal wastewater treatment plant introduced another geographic requirement for the facility - to be located near a road that allowed easy access to the containment tanks designed to capture all the wastewater for transport to a specialist decontamination facility.

Food waste, together with the surgical gowns worn by study staff when within the facility, was collected into medical waste containers. These containers were incinerated according to the local hospital protocol. Clothing worn by the volunteers was decontaminated the day before their departure from the facility using the chlorine dioxide gas, and on departure they wore the clothes they arrived in, which had been kept in the 'green zone'. In addition, tailored procedures needed to be established for the decontamination of the entire facility upon departure of all volunteers, intermittent decontamination of waste containers and of the belongings of the volunteers upon departure in a dedicated room within the facility. Decontamination with chlorine dioxide was again chosen for its characteristics as described above, but also as it leaves no post-decontamination residue, so all equipment (including sensitive electronics) could be left inside the facility during a decontamination cycle without risk of corrosion or other damage.

\section{Quality control \& assessment}

Operating within a contained environment necessitates preparations to prevent circumstances that might result in occupational injury, ill health or adverse environmental impact. In order to anticipate and prevent such circumstances, a structured approach was needed to identify hazards or forms of public health concern. Extensive health and environmental risk assessments were performed before the start of the study to determine the appropriate protective measures needed. Based on this assessment, SOPs were written including information about the hazards identified and how these can be mitigated. Personal safety measures, gowning procedures, waste management and decontamination procedures, accident prevention and contingency plans were among the most important potential hazards identified. In addition, a comprehensive communication plan was drafted with the support of the communication team of the University of Antwerp. Clearly, dealing with any incidents of potential virus escape via accidental release or need for emergency medical care of a vaccinated volunteer were among the most important risks to address and necessitated an emergency preparedness plan.

A specific concern of a clinical trial in a quarantine situation is that one must be prepared to adjust and adapt in case a volunteer has to leave a clinical trial at any point of time. The critical requirement in this context was the agreement to abide by a specially prepared SOP for anyone who left early so that they can be monitored with 
stool collection and testing until negative for viral shedding. In this case, arrangements were made for any potential early leavers to be accommodated in a local hotel in Antwerp where they would be provided with a chemical toilet to contain all stools and additional tailored guidance on hygienic measures, travel and contact restrictions. They were expected to continue to report to the study team with submission of stool samples on a daily basis. This only applied to one participant, who left on the last evening of their scheduled confinement but remained in the local area and participated in all final assessments on day 28.

\section{Lessons learned}

Planning and building a Phase I quarantine infrastructure like Poliopolis is a very challenging activity, considering the timing, global urgency for early data generation and specifications of containment. The whole concept allows collection of high-quality data and samples daily but requires total dedication and commitment for the duration of the study, from the study team as well as the volunteers. The study ran from 22 May to 22 August 2017, partly coinciding with the summer holiday period, creating an additional challenge to guarantee the permanent availability of nurses, coordinators and doctors by switching and shifting weekends and holidays. Volunteers went through a two-stage screening process, with medical and psychological assessments. The psychologists selected participants who would be able to cope with the constraints on an individual level, as well as to ensure each group of 15 volunteers, who did not know each other in advance, could function as a group. The psychological proofing appeared to be effective as there was a good spirit maintained in both groups throughout the study and any issues which arose were resolved through effective communication.

An inspection by the Regulatory Authorities in week 3 after the start of the Phase I study confirmed the high quality of the planning, preparedness, building facility and SOPs. The report of the clinical outcomes describes how the study was successfully completed by all enrolled individuals, with no dropouts, no issues with contamination and no evidence of any leaks of the candidate viruses [23]. Both vaccine candidates were well tolerated and immunogenic, and both were detected in the stools of the majority of participants, $100 \%$ after the first candidate and $87 \%$ after the second candidate. The median for cessation of shedding was 23 days (interquartile range [IQR]: 15-36) after the first candidate and 12 days (1-23) for the second candidate. More virus was shed by recipients of the first candidate than the second candidate, both as higher titers of and a longer duration of shedding. As reported, shed viruses from both candidates were found to be stable in terms of genetic sequence and reversion to neurovirulence [23].

This detailed description of the novel purpose-built contained facility illustrates the major effort by all concerned in planning and rapid implementation that were needed to achieve success in the Phase I novel polio vaccine study, allowing the analysis of the immunogenicity, safety and shedding. The clinical trial conducted in the facility described here was the first major step in the development of new OPVs in more than five decades. The steps followed in envisioning, planning and implementing the operational aspects of this study might be a model for future quarantine and human challenge vaccine experiments, and an important example for other projects performed as part of emergency vaccine development programs. New and evolving global initiatives such as the Coalition for Epidemic Preparedness Innovations (CEPI) recognise the urgency to respond to pandemic threats with rapid implementation of vaccine trials under containment [35].

\section{Summary}

The Poliopolis experience is the first of its kind, established in an unprecedented manner under the WHO containment recommendations (GAPIII) and with severe time constraints to implement an operationally challenging clinical trial with vaccine candidates that cannot yet be used under deliberate release conditions. The successful planning and implementation of this study should not only pave the way for rapid clinical development of the safer OPV formulations but should also provide a planning and contextual framework for future studies under containment to support global health initiatives such as those funded by CEPI in pandemic preparedness planning.

\section{Future perspective}

Although the global eradication of poliovirus type 2 was officially declared in September 2015, outbreaks of cVDPV2 still occur around the globe due to viral shedding from recipients of live OPVs. The development of the Poliopolis facility has been driven by the necessity to develop and test more genetically stable type 2 OPV for use in containing such outbreaks. Global withdrawal of live polioviruses has already started, with trivalent OPV being replaced by bivalent OPV which do not contain type 2 in May 2016. As the last case of type 3 poliovirus infection was reported in November 2012, one can anticipate the future declaration of eradication and withdrawal of type 
3 poliovirus from bivalent OPV, leaving only type 1, which is currently still being transmitted in three countries - Afghanistan, Pakistan and Nigeria. Once type 1 is eradicated, the use of OPV and global replacement with IPV would remove the risk of cVDPV and hence the requirement for genetically stable monovalent OPV (for outbreak use) and the Poliopolis facility (to test them).

Practice points

Need for novel vaccines

- Although wild poliovirus cases have virtually been eradicated there is an ongoing medical need for live vaccines to manage circulating vaccine-derived polioviruses (cVDPV) which are responsible for outbreaks in many countries.

- Current live vaccines are the source of CVDPV so new more genetically stable vaccines are required, particularly for type 2 poliovirus which has been declared to be globally eradicated.

- All oral poliovirus vaccines are shed in the stools of vaccinees to enter in the local environment, this being the source of cVDPV.

Rationale for containment

- Development of novel monovalent type 2 oral poliovirus vaccine in the current era of containment required a new approach to clinical testing.

- Following the global withdrawal of all live type 2 poliovirus vaccines (apart from stockpiles to manage cVDPV2 outbreaks) the GAPIII imposes stringent containment conditions for clinical testing of new vaccines to ensure minimal risks of environmental release by vaccine researchers and manufacturers.

- This Phase I study is the first to evaluate the stability of the novel genetically modified type 2 oral poliovirus vaccine candidates when administered to humans, so all precautions must be taken.

Planning

- The successful completion of the novel facility, Poliopolis, was the outcome of collaborative team effort involving medical, local and national government, and global health and regulatory authorities.

Building the infrastructure, 'Poliopolis'

- The facility was designed to accommodate 15 volunteers at any one time, with free access to cooking, entertainment and exercise facilities.

- An 'air lock' system allowed entry of study staff without risking release of study vaccine polioviruses.

External decontamination

- All potentially contaminated materials - waste washing and toilet water, food and clothing - were decontaminated using chlorine dioxide.

Quality control \& assessment

- Extensive health and environmental risk assessments were performed before the start of the study to determine the appropriate protective measures needed.

- As participants were to be confined for more than 28 days, psychological assessment of their suitability and compatibility with each other was essential.

- Regular monitoring of participants ensured they were happy to remain in the facility, helped by providing different forms of entertainment during the study.

\section{Acknowledgments}

The authors are grateful for the invaluable assistance of the many unnamed people mentioned without whose expert collaboration the creation and construction of Poliopolis would not have been possible, including the biosafety coordinator at Antwerp University Hospital, the psychologists and the other study staff. The authors thank RH Van Goethem and CEO B Heijnen (University of Antwerp) (and their staff), the Managing Director J Van der Straeten (Antwerp University Hospital) and the university hospital technical staff (D De Man and K Deckers) for making this project possible. They also thank K Veitch (keithveitch communications) for editorial assistance with the manuscript. The findings and conclusions in this report are those of the authors and do not necessarily represent the views of CDC and other contributing agencies.

The authors extend their thanks to PATH, the Bill \& Melinda Gates Foundation and PHARA for their continuous support, and to groep Arthur (Antwerp, Belgium) for assisting in project management.

Financial \& competing interests disclosure

Funding for the construction of Poliopolis, and the development and manufacture of the nOPV2 candidates was provided by the Bill \& Melinda Gates Foundation (grant number: OPP1149896 - title: New OPV Clinical Trials: Phase I studies). The authors have no other relevant affiliations or financial involvement with any organisation or entity with a financial interest in or financial conflict with the subject matter or materials discussed in the manuscript apart from those disclosed.

No writing assistance was utilized in the production of this manuscript. 


\section{Open access}

This work is licensed under the Creative Commons Attribution 4.0 License. To view a copy of this license, visit http://creativecomm ons.org/licenses/by/4.0/

\section{References}

Papers of special note have been highlighted as: • of interest $\bullet \bullet$ of considerable interest

1. Greene SA, Ahmed J, Datta SD et al. Progress toward polio eradication - Worldwide, January 2017-March 2019. MMWR Morb. Mortal. Wkly Rep. 68(20), 458-462 (2019).

-• The latest status update on global eradication of polioviruses.

2. Global Polio Eradication Initiative. Polio this week as of July 17: wild poliovirus type 1 and circulating vaccine-derived poliovirus cases. http://polioeradication.org/polio-today/polio-now/this-week/

3. Brouwer AF, Eisenberg JNS, Pomeroy CD et al. Epidemiology of the silent polio outbreak in Rahat, Israel, based on modeling of environmental surveillance data. Proc. Natl Acad. Sci. USA 115(45), E10625-E10633 (2018).

4. Wringe A, Fine PEM, Sutter RW, Kew OM. Estimating the extent of vaccine-derived poliovirus infection. PLoS ONE 10, e3433 (2008).

5. Kew $\mathrm{O}$, Morris-Glasgow V, Mauricio Landaverde $\mathrm{M}$ et al. Outbreak of poliomyelitis in Hispaniola associated with circulating type 1 vaccine-derived poliovirus. Science 296, 356-359 (2002)

6. Shimizu H, Thorley B, Paladin FJ et al. Circulation of type 1 vaccine-derived poliovirus in the Philippines in 2001. J. Virol. 78, $13,512-13,521$ (2004).

7. Arita M, Zhu SL, Yoshida H, Yoneyama T, Miyamura T, Shimizu H. A Sabin 3-derived poliovirus recombinant contained a sequence homologous with indigenous human enterovirus species $\mathrm{C}$ in the viral polymerase coding region. J. Virol. 79, 12,650-12,657 (2005).

8. Liang X, Zhang Y, Xu W et al. An outbreak of poliomyelitis caused by type 1 vaccine-derived poliovirus in China. J. Infect. Dis. 194, 545-551 (2006).

9. Rakoto-Andrianarivelo M, Gumede N, Jegouic S et al. Reemergence of recombinant vaccine-derived poliovirus outbreak in Madagascar. J. Infect. Dis. 197, 1427-1435 (2008).

10. Estívariz CF, Watkins MA, Handoko D et al. A large vaccine-derived poliovirus outbreak on Madura Island-Indonesia, 2005. J. Infect. Dis. 197, 347-354 (2008).

11. Grard G, Drexler JF, Lekana-Douki S et al. Type 1 wild poliovirus and putative enterovirus 109 in an outbreak of acute flaccid paralysis in Congo, October-November 2010. Euro Surveill. 15, pii:19723 (2010).

12. Mayor S. Polio outbreak in Ukraine likely to spread, WHO warns. BMJ 351, h4749 (2015).

13. Alleman MM, Chitale R, Burns CC et al. Vaccine-derived poliovirus outbreaks and events - three provinces, Demographic Republic of Congo, 2017. MMWR Morb. Mortal. Wkly Rep. 67(10), 300-305 (2018).

14. Bauri M, Wilkinson AL, Ropa B et al. Notes from the field: circulating vaccine-derived poliovirus type 1 and outbreak response - Papua New Guinea, 2018. MMWR Morb. Mortal. Wkly Rep. 68(5), 119-120 (2019).

15. Arie S. Polio virus spreads from Syria to Iraq. BMJ 348, g2481 (2014).

16. Eichner M, Brockmann SO. Polio emergence in Syria and Israel endangers Europe. Lancet. 382, 1777 (2013).

17. Hird TR, Grassly NC. Systematic review of mucosal immunity induced by oral and inactivated poliovirus vaccines against virus shedding following oral poliovirus challenge. PLoS Pathog. 8, e1002599 (2012).

18. Sáez-Llorens X, Clemens R, Leroux-Roels G et al. Immunogenicity and safety of a novel monovalent high-dose inactivated poliovirus type 2 vaccine in infants: a comparative, observer-blind, randomised, controlled trial. Lancet Infect. Dis. 16, 321-330 (2016).

19. Bandyopadhyay AS, Modlin JF, Wenger J, Gast C. Immunogenicity of new primary immunization schedules with inactivated poliovirus vaccine and bivalent oral polio vaccine for the polio endgame: a review. Clin. Infect. Dis. 67(Suppl. 1), S35-S41 (2018).

20. Fu R, Altamirano J, Sarnquist CC, Maldonado YA, Andrews JR. Assessing the risk of vaccine-derived outbreaks after reintroduction of oral poliovirus vaccine in postcessation settings. Clin. Infect. Dis. 67(Suppl. 1), S26-S34 (2018).

-. Key modelling data demonstrating the risk of circulating vaccine-derived poliovirus emergence following oral poliovirus vaccine $(\mathrm{OPV})$ use in the postcessation era.

21. Bandyopadhyay AS, Garon J, Seib K, Orenstein WA. Polio vaccination: past, present and future. Future Microbiol. 10(5), 791-808 (2015).

-. Comprehensive review of poliomyelitis, the virus, the vaccines and the programs.

22. Sutter RW. Unraveling the mucosal immunity of inactivated poliovirus vaccine. J. Infect. Dis. 217, 344-346 (2018).

23. Van Damme P, De Coster I, Bandyopadhyay AS et al. The safety and immunogenicity of two novel live attenuated monovalent (serotype 2) oral poliovirus vaccines in healthy adults: a double-blind, single-centre Phase I study. Lancet 394(10193), 148-158 (2019).

-. The report of the first clinical demonstration of novel OPV vaccine candidates in human volunteers - the first new OPV vaccines in over 50 years. 
24. WHO. WHO Global Action Plan to minimize poliovirus facility-associated risk after type-specific eradication of wild polioviruses and sequential cessation of oral polio vaccine use (GAPIII) - 2015.

WHO/POLIO/15.05.http://polioeradication.org/wp-content/uploads/2016/12/GAPIII_2014.pdf

25. Previsani N, Tangermann RH, Tallis G, Jafari HS. World Health Organization guidelines for containment of poliovirus following type-specific polio eradication-worldwide, 2015. MMWR Morb. Mortal. Wkly Rep. 64, 913-917 (2015).

- The WHO guidance for the global control of live poliovirus in the posteradication era.

26. Collett MS, Hincks JR, Benschop K et al. Antiviral activity of pocapavir in a randomized, blinded, placebo-controlled human oral poliovirus vaccine challenge model. J. Infect. Dis. 215(3), 335-343 (2017).

27. Lambkin-Williams R, Noulin N, Mann A, Catchpole A, Gilbert AS. The human viral challenge model: accelerating the evaluation of respiratory antivirals, vaccines and novel diagnostics. Respir. Res. 19(1), 123 (2018).

28. Onorato IM, Modlin JF, McBean AM, Thoms ML, Losonsky GA, Bernier R. Mucosal immunity induced by enhanced potency IPV and OPV. J. Infect. Dis. 163, 1-6 (1991).

29. Mohammed AJ, AlAwaidy S, Bawikar S et al. Fractional doses of inactivated poliovirus vaccine in Oman. N. Engl. J. Med. 362, 2351-2359 (2008).

30. Directive 2009/41/EC of the European Parliament and of the Council of 6 May 2009 on the contained use of genetically modified micro-organisms (Recast) Official Journal of the European Union L 125/75 -

21.5.2009. 75-97. https://eur-lex.europa.eu/LexUriServ/LexUriServ.do?uri=OJ:L:2009:125:0075:0097:EN:PDF

31. Roberts PV, Aieta EM, Berg JD, Chow BM. EPA-600/S2-81-092: Chlorine dioxide for wastewater disinfection: a feasibility evaluation (1981). https://nepis.epa.gov/Exe/ZyNET.exe/2000TBBZ.TXT?ZyActionD=ZyDocument\&Client=EPA\&Index=1981+Th $\mathrm{ru}+1985 \&$ Docs $=\&$ Query $=\&$ Time $=\&$ EndTime $=\&$ SearchMethod $=1 \&$ TocRestrict $=$ n $\&$ Toc $=\&$ TocEntry $=\& Q$ Field $=\& Q$ FieldYear $=\& Q$ FieldMonth $=\& Q$ FieldDay $=\& I n t Q F i e l d O p=0 \&$ ExtQFieldOp $=0 \& X m l Q u e r y=\&$ File $=D \% 3 A \% 5$ Czyfiles\%5CIndex\%20Data $\% 5 C 81$ th ru85\%5CTxt\%5C00000011\%5C2000TBBZ.txt\&User=ANONYMOUS\&Password=anonymous\&SortMethod=h\%7C-\&Maximum Documents=1\&Fuzzy Degree=0\&ImageQuality=r75g8/r75g8/x150y150g16/i425\&Display=hpf $\mathrm{r} \& D$ DefSeekPage $=\mathrm{x} \& S$ earchBack=ZyAc tionL\&Back=ZyActionS\&BackDesc=Results\%20page\&MaximumPages $=1 \& Z y$ Entry=1\&SeekPage $=x \& Z y P U R L$

32. Roberts PL, Lloyd D. Virus inactivation by protein denaturants used in affinity chromatography. Biologicals 35, 343-347 (2007).

33. WHO. Polio vaccines: WHO position paper - March 2016. Wkly Epidemiol. Rec. 91(12), 145-168 (2016).

-. Update on the situation of polio elimination in the world, with review of what is known up to 2016 and the WHO recommendations for polio vaccination.

34. Galazka A, Milstien J, Zaffran M. WHO/GPV/98.07: Thermostability of vaccines (1998). https://apps.who.int/iris/handle/10665/64980

35. Plotkin SA. Vaccines for epidemic infections and the role of CEPI. Hum. Vaccin. Immunother. 13(12), 2755-2762 (2017). 